\title{
Optimising and Recognising 2-Stage Delivery Chain with Time Windows
}

\author{
Frank Phillipson ${ }^{1}$, Max Ortega del Vecchyo ${ }^{12}$, Bart van Ginkel ${ }^{12}$, Dylan \\ Huizing $^{12}$, Alex Sangers ${ }^{1}$ \\ 1 TNO, PO Box 96800, 2509 JE The Hague, The Netherlands, \\ ${ }^{2}$ Delft University of Technology, The Netherlands
}

\begin{abstract}
In logistic delivery chains time windows are common. An arrival has to be in a certain time interval, at the expense of waiting time or penalties if the time limits are exceeded. This paper looks at the optimal placement of those time intervals in a specific case of a barge visiting two ports in sequence. For the second port a possible delay or penalty should be incorporated. Next, recognising these penalty structures in data is analysed. Do certain patterns in public travel data indicate that a certain dependency is existing.
\end{abstract}

Keywords: Logistics, Computation Models, 2-Stage Delivery, Data analysis

\section{Introduction}

Delivery windows are a known phenomenon in time window constrained models for production scheduling and vehicle routing. In [3] an overview can be found of recent literature on the use in production logistics. In the context of a delivery performance model, a delivery window is defined as the difference between the earliest acceptable delivery date and the latest acceptable delivery date. In supply chain management the problem of interest is the optimal positioning of the delivery time window to minimise the expected cost of untimely delivery, such as inventory costs and penalties or the estimation of accumulated delivery times with uncertainty $[4,3,5,6,7,9,10]$.

Delivery windows are also used in Vehicle Routing Problems (VRP). A VRP involves finding a set of routes, starting and ending at a depot, that together cover a set of customers. Each customer has a given demand, and no vehicle can service more customers than its capacity permits. The objective is to minimise the total distance travelled or the number of vehicles used, or a combination of these. A special case of the VRP is when the service at a customer's place must start within a given time window. There are two types of time windows. Time windows are called soft when they can be violated for a penalty cost. They are hard when they cannot be violated, i.e., if a vehicle arrives too early at a customer, it must wait until the time window opens; and it is not allowed to 
arrive late. In all the cases these time windows are given in advance $[8,2,1]$.

In this work a delivery chain is studied where a barge has to visit two ports. In each port a number of containers is handled. For the planning of the port, the planner of the barge should indicate a time slot in which the barge will arrive. If the barge is too early, it has to wait until the beginning of the slot. If the barge is too late, it has to wait some penalty time. If the barge arrives within the time slot, the handling starts immediately. This means that we introduce a penalty which occurrence is dependent on the arrival time, which duration is dependent on the arrival time in case of early arrival, in combination with a two-stage time window. Within this study, first the optimisation of the choice of the time slots is elaborated in Section 2. The main question here is what the optimal time slots are to be communicated to minimise the total of the penalties. Secondly, in Section 3 the way to recognise the existence of such time slots with penalties in travel data is studied. In practice often not all data and/or the precise process is known. There the question is if we only see the arrival and departure times of a barge (for example from GPS or AIS data) can we predict the underlying process, to be able to predict the arrival time of the barges at some (final) stop.

\section{Optimisation}

The central case in this paper is a delivery chain where a barge has to visit two ports. In each port a number of containers should be handled. For the planning of the port, the planner of the barge should indicate a time slot in which the barge will arrive. If the barge is too early, it has to wait until the beginning of the slot. If the barge is too late, it has to wait some penalty time. If the barge arrives within the time slot, the handling starts immediately. In this section the optimal choice of the time window is determined.

\subsection{Problem description}

To formulate the problem, first some notation is defined:

$T_{i}=$ Transportation time to port $i$, starting at the former location;

$H_{i}=$ Handling time at port $i$;

$W_{i}=$ Waiting time at port $i$

$S_{i}=$ Start time slot at port $i$

$L=$ Length time slot

$K=$ Penalty wait time

The question that arises is what would be the optimal start times of both slots $\left(S_{1}\right.$ and $\left.S_{2}\right)$ to minimise the sum of the waiting times $\left(W_{1}+W_{2}\right)$ ? Different probability distribution functions are used for the transportation and handling times and, as a consequence, for the arrival time $(X)$ at the port under consideration. The arrival, in each of the ports therefor we skip the indices here, will be in the interval $(A, B)$ (see Fig. 1). We assume that, for each of the two stages, 
$S \geq A$ and $B \geq S+L$, while losing a part of the time slot will not be smart. Only if $L \geq(B-A)$ this will not hold, but then we have no problem. The arrival will be in one of the three intervals $a, b$ or $c$. For each realisation of the arrival time $x$ we can calculate the waiting time:

$$
\begin{aligned}
& A \leq x<S \quad W=S-x \\
& S \leq x \leq S+L \quad W=0 \\
& S+L<x \leq B \quad W=K
\end{aligned}
$$

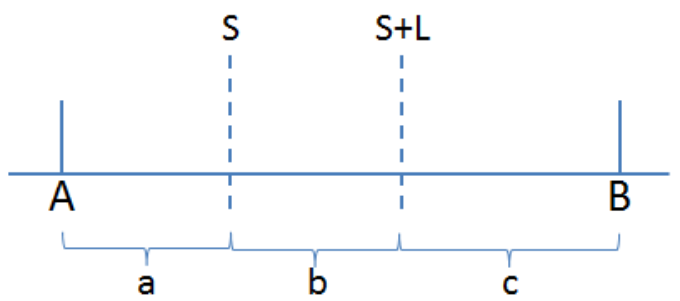

Fig. 1. Process

\section{$2.2 \quad$ First stage}

Now the optimal choice for the starting time of the time slots can be derived, by minimising the expected waiting time as a function of $S_{1}$. We assume three different options for the penalty: a fixed time, a function of the delay and a random value. At the first port the arrival time $X$ is equal to the transportation time $T_{1}$. For various probability distribution functions for $T_{1}$ we obtain the optimal value $(S)$ for $S_{1}$, the start of the first time slot.

Fixed penalty Given a fixed penalty $K$, the expected waiting time is given by:

$$
\begin{aligned}
\mathbb{E}[W] & =\mathbb{E}\left[W \mathbb{1}_{X<S}\right]+\mathbb{E}\left[W \mathbb{1}_{S \leq X<S+L}\right]+\mathbb{E}\left[W \mathbb{1}_{S+L \leq X}\right] \\
& =\mathbb{E}\left[(S-X) \mathbb{1}_{X<S}\right]+\mathbb{E}\left[0 \mathbb{1}_{S \leq X<S+L}\right]+\mathbb{E}\left[K \mathbb{1}_{S+L \leq X}\right] \\
& =S \mathbb{E}\left[\mathbb{1}_{X<S}\right]-\mathbb{E}\left[X \mathbb{1}_{X<S}\right]+0+K \mathbb{E}\left[\mathbb{1}_{S+L \leq X}\right] \\
& =S F(S)-\int_{-\infty}^{\infty} x \mathbb{1}_{x<S} f(x) d x+K(1-F(S+L)) \\
& =S F(S)-\int_{-\infty}^{S} x f(x) d x+K(1-F(S+L)) .
\end{aligned}
$$

The expected waiting time is minimised by:

$$
\frac{d}{d S} \mathbb{E}[W]=0
$$


resulting in

$$
\begin{aligned}
\frac{d}{d S} \mathbb{E}[W] & =\frac{d}{d S} S F(S)-\frac{d}{d S} \int_{-\infty}^{S} x f(x) d x+\frac{d}{d S} K(1-F(S+L)) \\
& =S f(S)+F(S)-S f(S)-K f(S+L)=F(S)-K f(S+L)
\end{aligned}
$$

So:

$$
\frac{d}{d S} \mathbb{E}[W]=0 \Longleftrightarrow F(S)=K \cdot f(S+L)
$$

Now any distribution for $X$ can be used. For three examples this will be elaborated.

Uniform distribution If the transportation time and consequently the arrival time $X$ is uniform $(\mathrm{A}, \mathrm{B}): F(S)=\frac{S-A}{B-A}$ and $f(S)=\frac{1}{B-A}$, so we obtain:

$$
\frac{d}{d S} \mathbb{E}[W]=0 \Longleftrightarrow \frac{S-A}{B-A}=K \cdot \frac{1}{B-A} \Longleftrightarrow S-A=K \Longleftrightarrow S=A+K .
$$

Recall that $S$ has a maximum value of $B-L$, thus $S=\min (A+K, B-L)$.

Exponential distribution If the transportation time and consequently the arrival time $X$ is exponential distributed $(\lambda)$ the expected waiting time equals: Exponential: $F(S)=1-\exp ^{-\lambda S}$ and $f(S)=\lambda \exp ^{-\lambda S}$. So we obtain:

$$
\begin{aligned}
\frac{d}{d S} \mathbb{E}[W]=0 & \Longleftrightarrow 1-\exp ^{-\lambda S}=K \lambda \exp ^{-\lambda(S+L)} \\
& \Longleftrightarrow 1=\left(K \lambda \exp ^{-\lambda L}+1\right) \exp ^{-\lambda S} \\
& \Longleftrightarrow-\lambda S=\log \left(\frac{1}{K \lambda \exp ^{-\lambda L}+1}\right) \\
& \Longleftrightarrow S=\frac{1}{\lambda} \log \left(K \lambda \exp ^{-\lambda L}+1\right)
\end{aligned}
$$

Normal distribution If the arrival time is normal $(\mu, \sigma)$ distributed, where $\phi($.$) denotes the normal probability density function and \Phi($.$) the cumulative$ probability density, the waiting time is minimised by solving for $S$ in:

$$
\Phi(S)=K \phi(S+L),
$$

which has to be solved numerically.

Penalty as function of delay Now assume the penalty depends on how late the barge is. Again, $\mathbb{E}[W]$ is calculated, since the only term that changes compared to the situation above is $\mathbb{E}\left[W \mathbb{1}_{S+L \leq X}\right]$. The penalty equals $k(X-S-L)$ for some function $k:[0, \infty) \rightarrow[0, \infty)$.

$$
\mathbb{E}\left[W \mathbb{1}_{S+L \leq X}\right]=\mathbb{E}\left[k(X-S-L) \mathbb{1}_{S+L \leq X}\right]=\int_{S+L}^{\infty} k(x-S-L) f(x) d x
$$


The derivative follows from ${ }^{3}$ :

$$
\begin{aligned}
\frac{d}{d S} \mathbb{E}\left[W \mathbb{1}_{S+L \leq X}\right] & =\frac{d}{d S} \int_{S+L}^{\infty} k(x-S-L) f(x) d x \\
& =\int_{S+L}^{\infty}-k^{\prime}(x-S-L) f(x) d x-\left.k(x-S-L) f(x)\right|_{x=S+L} \\
& =-\int_{S+L}^{\infty} k^{\prime}(x-S-L) f(x) d x-k(0) f(S+L) \\
& =-\int_{0}^{\infty} k^{\prime}(x) f(x+S+L) d x-k(0) f(S+L)
\end{aligned}
$$

Combining with the steps above results in:

$$
\frac{d}{d S} \mathbb{E}(W)=F(S)-\int_{0}^{\infty} k^{\prime}(x) f(x+S+L) d x-k(0) f(S+L)
$$

So

$$
\frac{d}{d S} \mathbb{E}(W)=0 \Longleftrightarrow F(S)=\int_{0}^{\infty} k^{\prime}(x) f(x+S+L) d x+k(0) f(S+L)
$$

Note that if $k$ is a constant, this expression reduces to what was found earlier.

Penalty is random variable, independent of $\mathbf{X}$ The third option concerns a random penalty $K$, independent of $X$. Then last term becomes:

$$
\mathbb{E}\left[W \mathbb{1}_{S+L \leq X}\right]=\mathbb{E}\left[K \mathbb{1}_{S+L \leq X}\right]
$$

Since $K$ and $X$ are independent, so are $K$ and $\mathbb{1}_{S+L \leq X}$, the expectations can be multiplied to obtain:

$$
\begin{gathered}
\mathbb{E}\left[W \mathbb{1}_{S+L \leq X}\right]=\mathbb{E}[K] \mathbb{E}\left[\mathbb{1}_{S+L \leq X}\right]=\mathbb{E}[K](1-F(S+L)) \\
\frac{d}{d S} \mathbb{E}(W)=0 \Longleftrightarrow F(S)=\mathbb{E}[K] f(S+L)
\end{gathered}
$$

In the case that $K$ is constant this expression reduces to the first case again.

\subsection{Second stage}

The first stage resulted in a general formulation that can be used for the second stage, given that the probability distribution of the arrival time at the second port is known. However, the probability distribution function of the arrival time $(X)$ is more complicated, namely the sum of two transportation times, a handling time and possibly a penalty. In Section 2.3 the penalty is neglected, later, in Section 2.3 , the propagation of the penalty is studied.

\footnotetext{
${ }^{3}$ Under some regularity assumptions, for instance $k$ must be differentiable on $(0, \infty)$
} and continuous on $[0, \infty)$ ) 
Second time slot without penalty in the first time slot For the second time slot without penalty, the same approach can be taken as in the first stage. First note that here it is assumed that there is no penalty in the first time slot, but obviously there is one in the second (since otherwise nothing would have to be optimised). Now again for the three probability distributions (of each of the stochastic variables, adding up to the arrival time at the second stage) the solution can be derived.

Uniform distribution If the two transportation times and the handling time all follow a uniform distribution, the arrival time has an Irwin-Hall distribution. This distribution converges quickly to the normal distribution. From our experience, even in the case of only three underlying uniform distributions a normal approximation is usable in practice. If $T_{1} \sim$ uniform $\left(U_{1}, U_{2}\right), T_{2} \sim$ uniform $\left(U_{3}, U_{4}\right)$ and $H_{1} \sim$ uniform $\left(U_{5}, U_{6}\right)$ then by approximation $X \sim$ $\operatorname{Normal}(\mu, \sigma)$ where

$$
\begin{gathered}
\mu=\frac{1}{2}\left(U_{2}-U_{1}\right)+\frac{1}{2}\left(U_{4}-U_{3}\right)+\frac{1}{2}\left(U_{6}-U_{5}\right), \\
\sigma=\sqrt{\frac{\left(U_{2}-U_{1}\right)^{2}}{12}+\frac{\left(U_{4}-U_{3}\right)^{2}}{12}+\frac{\left(U_{6}-U_{5}\right)^{2}}{12} .}
\end{gathered}
$$

Now the method for the normal distribution of the previous stage can be used.

Exponential distribution In the case of exponential handling and transporting times (and assuming independence) the second arrival time has an Erlang $(3, \lambda)$ distribution. This means:

$$
\begin{aligned}
& F(x)=1-\sum_{n=0}^{2} \frac{1}{n !} \exp ^{-\lambda x}(\lambda x)^{n} \\
& f(x)=\frac{1}{2} \lambda^{3} x^{2} \exp ^{-\lambda x}
\end{aligned}
$$

The formula above reduces the problem to finding $s$ such that:

$$
\begin{aligned}
& 1-\exp ^{-\lambda S}-\lambda S \exp ^{-\lambda S}-\frac{1}{2} \lambda^{2} S^{2} \exp ^{-\lambda S}=\frac{1}{2} K \lambda^{3} S^{2} \exp ^{-\lambda(S+L)} \\
\Longleftrightarrow & \exp ^{\lambda S}-1-\lambda S-\frac{1}{2} \lambda^{2} S^{2}=\frac{1}{2} K \lambda^{3} S^{2} \exp ^{-\lambda L} \\
\Longleftrightarrow & \exp ^{\lambda S}=\frac{1}{2}\left(K \lambda^{3} \exp ^{-\lambda L}+\lambda^{2}\right) S^{2}+\lambda S+1 .
\end{aligned}
$$

The latter expression can be solved for $S$ numerically.

Normal distribution If the two transportation times and the handling time are all normally distributed and independent, the arrival time has a again a normal distribution. If $T_{1} \sim \operatorname{Normal}\left(\mu_{1}, \sigma_{1}\right), T_{2} \sim \operatorname{Normal}\left(\mu_{2}, \sigma_{2}\right)$ and $H_{1} \sim$ $\operatorname{Normal}\left(\mu_{3}, \sigma_{3}\right)$ then $X \sim \operatorname{Normal}(\mu, \sigma)$ where

$$
\mu=\mu_{1}+\mu_{2}+\mu_{3}
$$




$$
\sigma=\sqrt{\sigma_{1}^{2}+\sigma_{2}^{2}+\sigma_{3}^{2}} .
$$

Now the method for the normal distribution of the previous section can be used.

Propagation of penalty: Second time slot with penalty The challenge now is to derive an expression for the arrival time at the second port, including the fact that there may have been a penalty at the first port. Then the formula presented earlier can be applied to find the expression that has to be solved.

We assume $T_{1}, T_{2}$ and $H_{1}$ to be independent. The time that is added to this due to not arriving within the time frame, is the penalty $P$. So $P$ is not only due to arriving late. We see then:

$$
P=\left\{\begin{array}{llr}
S-T_{1} & \text { if } & T_{1} \leq S \\
0 & \text { if } & S<T_{1} \leq S+L \\
k & \text { if } & T_{1}>S+L
\end{array}\right.
$$

Now we are interested in the second arrival time $X=T_{1}+P+H+T_{2}$. Since $P$ and $T_{1}$ are dependent of each other and the rest is independent, we will call $X_{1}=T_{1}+P$ and $X_{2}=H+T_{2}$. The interesting part here is $X_{1}$ :

$$
\begin{array}{r}
X_{1}=\left\{\begin{array}{lrr}
T_{1}+S-T_{1} & \text { if } & T_{1} \leq S \\
T_{1}+0 & \text { if } & S<T_{1} \leq S+L \\
T_{1}+k & \text { if } & T_{1}>S+L
\end{array}\right. \\
=\left\{\begin{array}{llr}
S & \text { if } & T_{1} \leq S \\
T_{1} & \text { if } & S<T_{1} \leq S+L \\
T_{1}+k & \text { if } & T_{1}>S+L
\end{array}\right.
\end{array}
$$

Now the cumulative distribution function of $X_{1}$ equals:

$$
F_{X_{1}}(x)=\left\{\begin{array}{llr}
0 & \text { if } & x<S \\
F_{T_{1}}(x) & \text { if } & S \leq x \leq S+L \\
F_{T_{1}}(S+L) \text { if } & S+L<x \leq S+L+k \\
F_{T_{1}}(x-k) \text { if } & x>S+L+k
\end{array}\right.
$$

This is visualised in Fig. 2. The jump in the point $S$ means that the random variable is not absolutely continuous. This is what we expect, since the probability of starting the handling at point $S$ equals $\mathbb{P}\left(T_{1} \leq S\right)=F_{T_{1}}(S)$, which is strictly positive. We can describe the 'density' in this way:

$$
f_{X_{1}}(x)=\left\{\begin{array}{llr}
0 & \text { if } & x<S \\
\operatorname{mass} F_{T_{1}}(S) \text { if } & x=S \\
f_{T_{1}}(x) & \text { if } & S \leq x \leq S+L \\
0 & \text { if } & S+L<x \leq S+L+k \\
f_{T_{1}}(x-k) & \text { if } & x>S+L+k
\end{array}\right.
$$

Now we would like to obtain the cumulative density function and the density of 


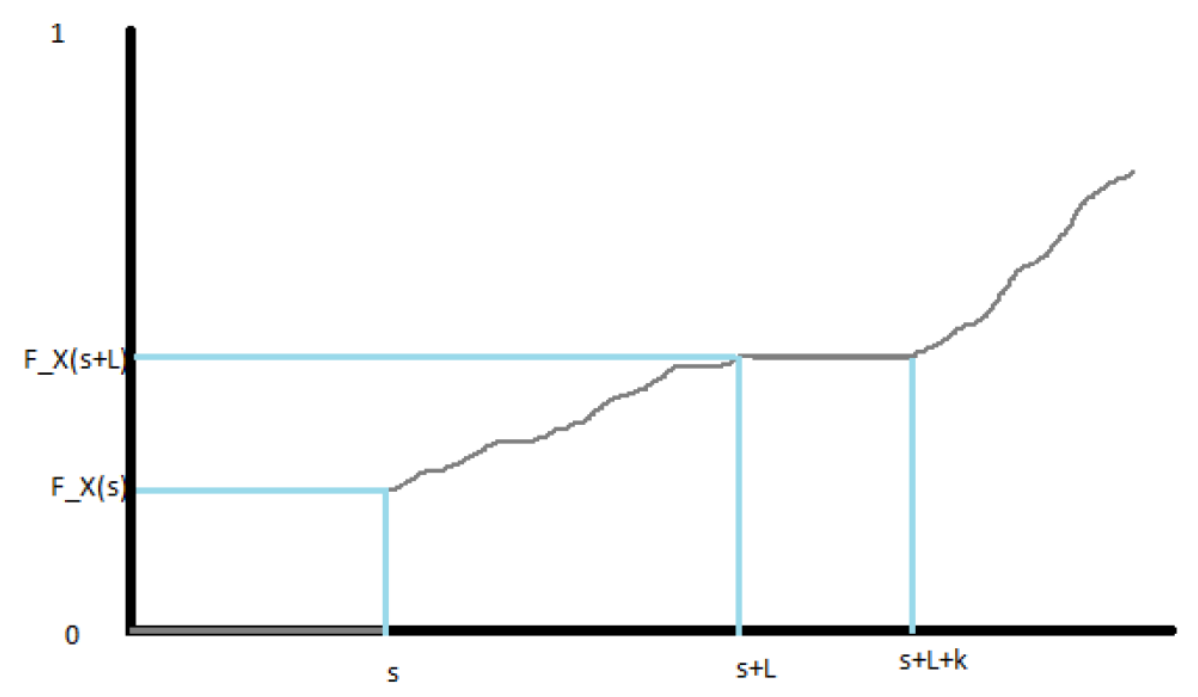

Fig. 2. CDF of B: time that handling begins

the sum of $X_{1}$ and $X_{2}{ }^{4}$.

$$
\begin{aligned}
F_{X}(x) & =\mathbb{P}\left(X_{1}+X_{2} \leq x\right)=\int_{b=-\infty}^{\infty} \int_{a=-\infty}^{x-b} f_{X_{1}, X_{2}}(b, a) \mathrm{d} a \mathrm{~d} b \\
& =\int_{b=-\infty}^{\infty} \int_{a=-\infty}^{x-b} f_{X_{1}}(b) f_{X_{2}}(a) \mathrm{d} a \mathrm{~d} b=\int_{b=-\infty}^{\infty} \int_{a=-\infty}^{x-b} f_{X_{2}}(a) \mathrm{d} a f_{X_{1}}(b) \mathrm{d} b \\
& =\int_{-\infty}^{\infty} F_{X_{2}}(x-b) f_{X_{1}}(b) \mathrm{d} b
\end{aligned}
$$

Now, using the description that we found of $f_{X_{1}}$, we obtain:

$$
\begin{aligned}
F_{X}(x)= & \int_{-\infty}^{\infty} F_{X_{2}} A(x-b) f_{X_{1}}(b) \mathrm{d} b \\
= & F_{T_{1}}(S) F_{X_{2}}(x-S)+\int_{S}^{S+L} F_{X_{2}}(x-b) f_{T_{1}}(b) \mathrm{d} b \\
& +\int_{S+L+k}^{\infty} F_{X_{2}}(x-b) f_{T_{1}}(b-k) \mathrm{d} b
\end{aligned}
$$

\footnotetext{
${ }^{4}$ Note that the following computations are strictly speaking ill-defined, since $f$ is not a continuous function. However, it is correct and this way a more intuitive derivation is given. To be precise, one would have to use the Lebesgue-Stieltjes integral to avoid speaking of $f$. Also note that we use independence of $X_{1}$ and $X_{2}$ when their joint probability distribution function is written as the product of the marginals.
} 
Differentiating this with respect to $x$ yields (under some regularity conditions):

$$
\begin{aligned}
f_{X}(x)= & F_{T_{1}}(S) f_{X_{2}}(x-S)+\int_{S}^{S+L} f_{X_{2}}(x-b) f_{T_{1}}(b) \mathrm{d} b \\
& +\int_{S+L+k}^{\infty} f_{X_{2}}(x-b) f_{T_{1}}(b-k) \mathrm{d} b
\end{aligned}
$$

Note that $X_{2} \geq 0$, so $f_{X_{2}}(x-b)$ will be 0 for $b>x$. So in practice, a part of the integral will drop out.

To find the optimal time, we need to use the formula of the first stage optimisation again: $F_{X}\left(S_{2}\right)=k_{2} f_{X}\left(S_{2}+L_{2}\right)$. We obtain as the equation that has to be solved for $S_{2}$ :

$$
\begin{aligned}
& F_{T_{1}}(S) F_{X_{2}}\left(S_{2}-S\right)+\int_{S}^{S+L} F_{X_{2}}\left(S_{2}-b\right) f_{T_{1}}(b) \mathrm{d} b \\
& +\int_{S+L+k}^{\infty} F_{X_{2}}\left(S_{2}-b\right) f_{T_{1}}(b-k) \mathrm{d} b=k_{2} F_{T_{1}}(S) f_{X_{2}}\left(S_{2}+L_{2}-S\right) \\
& +k_{2} \int_{S}^{S+L} f_{X_{2}}\left(S_{2}+L_{2}-b\right) f_{T_{1}}(b) \mathrm{d} b+k_{2} \int_{S+L+k}^{\infty} f_{X_{2}}\left(S_{2}+L_{2}-b\right) f_{T_{1}}(b-k) \mathrm{d} b
\end{aligned}
$$

By rearranging a bit, we get:

$$
\begin{aligned}
& \left(k_{2} f_{X_{2}}\left(S_{2}+L_{2}-S\right)-F_{X_{2}}\left(S_{2}-S\right)\right) F_{T_{1}}(S) \\
= & \int_{S}^{S+L}\left(F_{X_{2}}\left(S_{2}-b\right)-k_{2} f_{X-2}\left(S_{2}+L_{2}-b\right)\right) f_{T_{1}}(b) \mathrm{d} b \\
+ & \int_{S+L+k}^{\infty}\left(F_{X_{2}}\left(S_{2}-b\right)-k_{2} f_{X_{2}}\left(S_{2}+L_{2}-b\right)\right) f_{T_{1}}(b-k) \mathrm{d} b
\end{aligned}
$$

Note that in any situation with a sum of random variables, the convolution integral appears. This usually cannot be simplified, except for nice situations such as some known sums of random variables. This is the reason for the integrals with two densities in them. The penalty $P$ is of different nature in different cases, this accounts for the multiple integrals. This suggests that there is not much hope of finding nicer expressions.

\subsection{Case}

As example we look at the following case. As input data we use:

$$
\begin{aligned}
& T_{1}=U(180 ; 234) \\
& H_{1}=U(50 ; 150) \\
& T_{2}=U(120 ; 156) \\
& H_{2}=U(50 ; 150) \\
& L=30 \text { minutes } \\
& K=45 \text { minutes }
\end{aligned}
$$


Now the optimal value of $S_{1}$ can be calculated by $S_{1}^{*}=\min (A+P, B-L)=$ $\min (180+45 ; 234-30)=204$ resulting in $E\left(W_{1}\right)=5.33$. The same for $S_{2}$. First for the case neglecting the penalty at the first port. Minimum value for $S_{2}$ can be derived easily $S_{2}=180+50+120=350$, and also the maximum value $S_{2}=234+150+156=540$. The arrival time on port 2 is a sum of three uniform distributed variables. If we assume that the sum of three uniform variables has a normal distribution, then the arrival time on port 2 is normal distributed with $\mu=350+0.5 *(190)=445$ and $\sigma=\sqrt{\frac{(54)^{2}}{12}+\frac{(100)^{2}}{12}+\frac{(36)^{2}}{12}}=34.4$. Solving the formula of the first stage for a normal distribution gives $S_{2}^{*}=438$.

These results can be checked by a numerical simulation of 70,000 realisations of trips with these parameters. Figure 3 shows that the minimum delay is reached (indeed) around 204. Also it can be seen how sensitive the outcome is for a choice of $S_{1}$ : 10 minutes off, gives 5 minutes extra delay.

Simulating the second stage without penalty results in the outcome as shown in Fig. 4. The optimal value of 438 is confirmed, however the graph is rather flat around the optimum, and the sensitivity of the delay on the window is low.

From the simulation of the second stage with penalty at the first stage also comes that taking the penalty into account the optimal $S_{2} *$ becomes 441 , as depicted in Fig. 5.

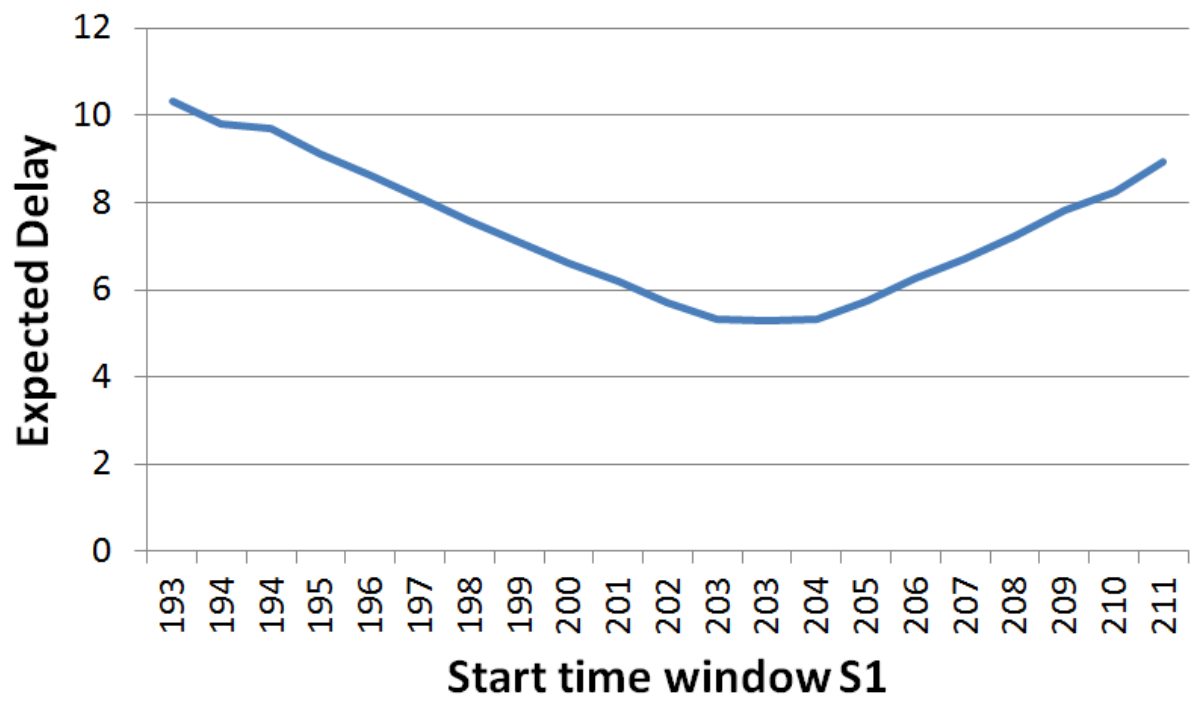

Fig. 3. Simulated optimum $S_{1}$. 


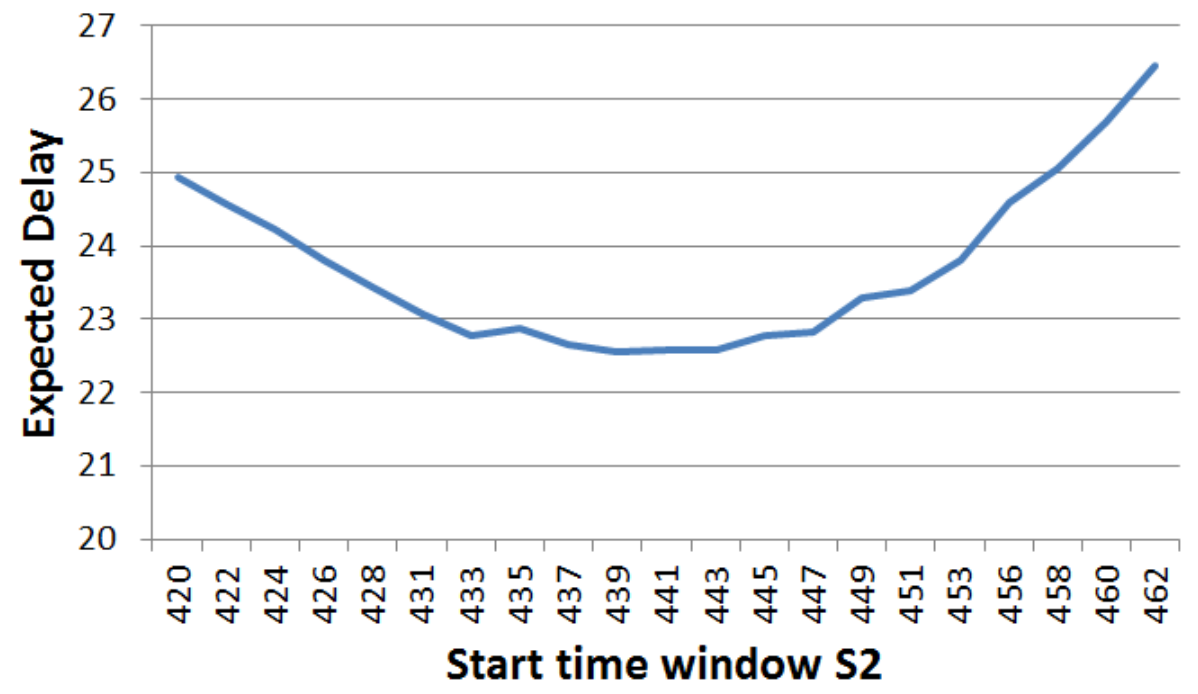

Fig. 4. Simulated optimum $S_{2}$ without penalty.

\section{Recognizing time windows in data}

In practice often not all data and/or the precise process is known. For example only GPS-data is available and this information is used in planning. Then it would be nice to understand where the interactions and (possible) correlations in data comes from. In this section we look at the case were we only see the arrival and departure times of a barge (for example from GPS or AIS data) and want to understand the underlying process better by analysing this data. We want, for example, to be able to predict the arrival time of the barges at some (final) stop. For this we can try to predict the separate steps in the chain, here for example the transportation times and the handling times. But what if there are dependencies, for example caused by waiting times that are depending on whether some time slot is met by arrival, as explained in the previous section.

\subsection{Analysis}

To get some idea on this, we simulated the process as defined in the previous section for four cases:

1. No time slot; a barge is handled on arrival at each port;

2. Optimal time slots chosen; as defined in the previous section;

3. Time slots are chosen around the expected arrival time; the planner puts the time slot symmetrically around the expected arrival time;

4. No optimisation; the planner places the start of the time slot at the earliest arrival time. 


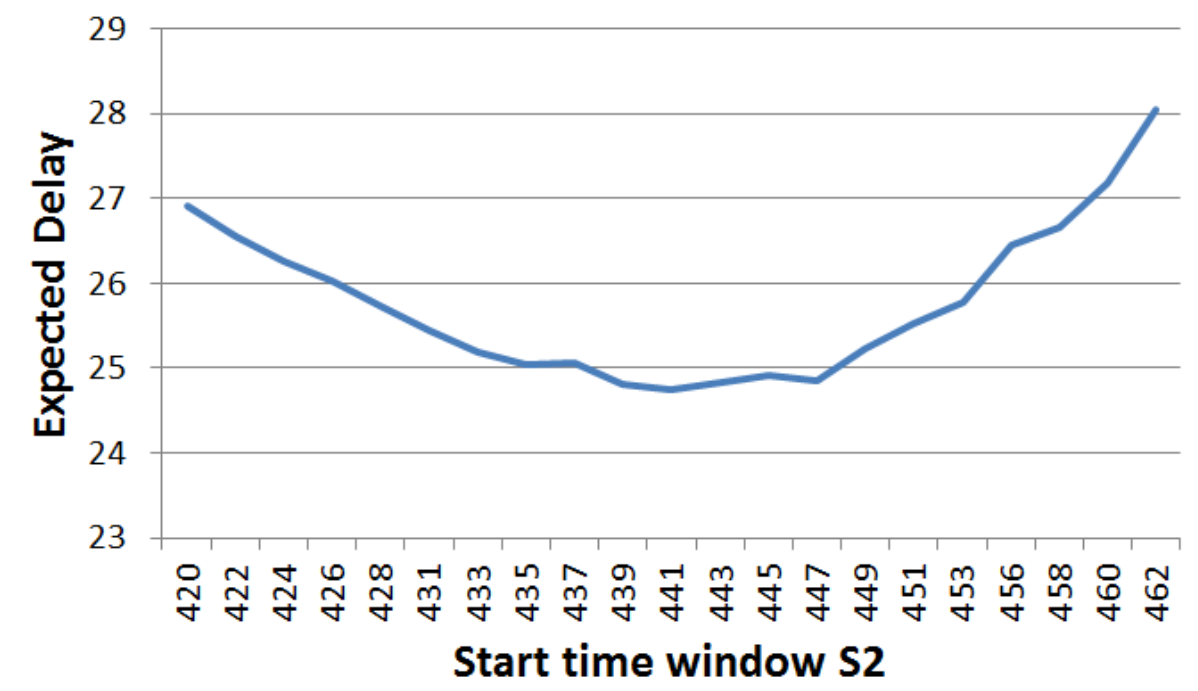

Fig. 5. Simulated optimum $S_{2}$ with penalty.

As numerical input we take (in minutes):

$$
\begin{aligned}
& T_{1}=U(180 ; 234) \\
& H_{1}=U(50 ; 150) \\
& T_{2}=U(120 ; 156) \\
& H_{2}=U(50 ; 150) \\
& L=30 \\
& K=30
\end{aligned}
$$

This gives a minimal lead time of 400 minutes and a maximum lead time of 690 (plus 60 minutes of penalties) minutes. For each of the four cases we simulated 5,000 realisations, were only the arrival and departure times were reported. From these times the two transportation and two handling times were calculated, as depicted in Fig. 6. Again for each of the four cases, the correlation between the four arrival/departure times and the average total lead time were derived. For each correlation value also the p-value was calculated to test whether the correlation is significantly different from zero. The results are presented in Table 1 until Table 5.

Table 1. Correlation in the case 'No time slot'; p-value between brackets.

\begin{tabular}{lccc}
\hline & $T_{1}$ & $H_{1}$ & $T_{2}$ \\
\hline$H_{1}$ & $0.028(0.052)$ & & \\
$T_{2}$ & $-0.018(0.201)$ & $0.001(0.961)$ & \\
$H_{2}$ & $0.022(0.122)$ & $-0.005(0.750)$ & $-0.001(0.952)$ \\
\hline
\end{tabular}


Table 2. Correlation in the case 'Optimal time slot'; p-value between brackets.

\begin{tabular}{|c|c|c|}
\hline \multirow{2}{*}{$\begin{array}{c}T_{1} \\
H_{1}-0.210(0.000)\end{array}$} & $H_{1}$ & $T_{2}$ \\
\hline & & \\
\hline$T_{2} \quad 0.004(0.787)$ & $0.003(0.850)$ & \\
\hline$H_{2}-0.008(0.559)$ & $-0.056(0.000)$ & $-0.005(0.741)$ \\
\hline
\end{tabular}

Table 3. Correlation in the case 'Time slots around the expected arrival time'; p-value between brackets.

\begin{tabular}{llcc}
\hline & $T_{1}$ & $H_{1}$ & $T_{2}$ \\
\hline$H_{1}$ & $0.216(0.000)$ & & \\
$T_{2}$ & $0.008(0.562)$ & $-0.012(0.406)$ & \\
$H_{2}$ & $0.047(0.000)$ & $0.063(0.000)$ & $-0.009(0.518)$ \\
\hline
\end{tabular}

Table 4. Correlation in the case 'Not optimised'; p-value between brackets.

\begin{tabular}{|c|c|c|}
\hline$T_{1}$ & $H_{1}$ & $T_{2}$ \\
\hline $\begin{array}{ll}H_{1} & 0.382(0.000)\end{array}$ & & \\
\hline$T_{2}-0.007(0.635)$ & $-0.011(0.431)$ & \\
\hline$H_{2} \quad 0.171(0.000)$ & $0.232(0.000)$ & $0.031(0.0278)$ \\
\hline
\end{tabular}

Table 5. Total process time of the four cases.

\begin{tabular}{|c|c|}
\hline \multicolumn{2}{|c|}{ Case Total time } \\
\hline 1 & 545 \\
\hline 2 & 567 \\
\hline 3 & 572 \\
\hline 4 & 581 \\
\hline
\end{tabular}




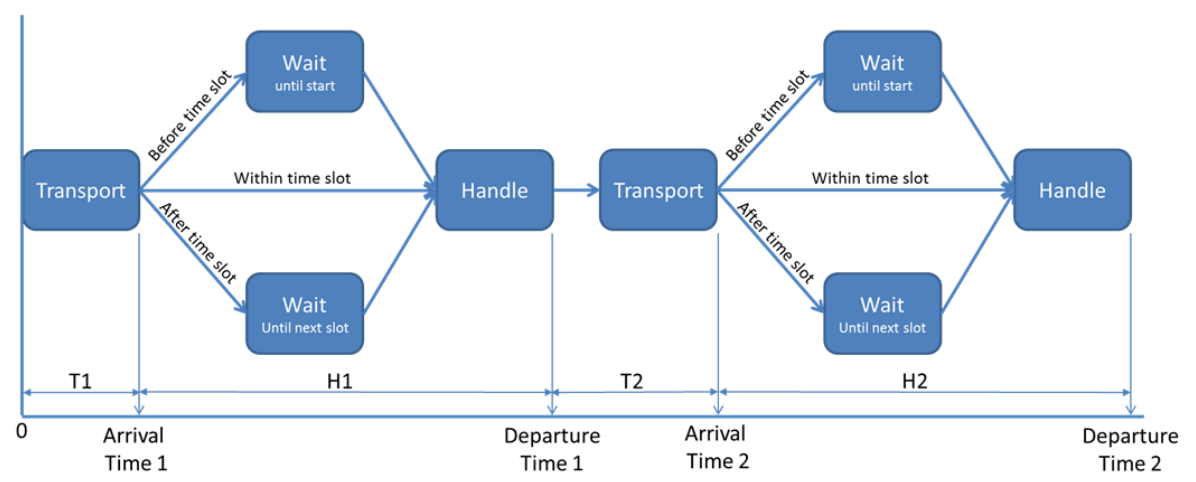

Fig. 6. Process

There are some observations we can make:

1. In the case 'No time slots' there is no correlation between the transportation and handling times.

2. In the case 'No optimisation' there exist: positive correlation between $\left(T_{1}\right.$, $\left.H_{1}\right)$, positive correlation between $\left(T_{1}, H_{2}\right)$ and positive correlation between $\left(H_{1}, H_{2}\right)$, all by the penalty. There also is a (small but significant) correlation between $\left(T_{2}, H_{2}\right)$.

3. In the case 'Time slots are chosen around the expected arrival time', the correlations become lower; the effect of the penalty is less than in the not optimised case.

4. In the case 'Optimal time slots chosen', the correlation between $\left(T_{1}, H_{2}\right)$ disappear (no delay propagation anymore), the two other relations that had a positive correlation $\left(T_{1}, H_{1}\right)$ and $\left(H_{1}, H_{2}\right)$ become negative. This means that longer delays do not cause the big penalty anymore, but being early (lower arrival time) leads to small waiting times.

\subsection{Limitations}

Up to here we assumed that the planning and realisations are independent. However in practice people are going to react on realisations. For example:

- If the barge is early, the captain can decide to slow down and save fuel. This could lead to a shift in the transportation time distribution and from the optimised case to the 'time slots around expected arrival time' case.

- If the barge had delay in the first part (transportation, penalty and/or handling) the captain could decide to go faster. This again leads to a shift in the transportation time distribution and potentially a decrease in the correlation between $\left(T_{1}, T_{2}\right)$ and $\left(H_{1}, T_{2}\right)$. 


\section{Conclusions}

This paper looked at a delivery chain in logistics, where a barge has to visit two ports and was faced by delivery time slot in which the barge has to arrive. We looked at two issues; first how can the time slot be chosen optimally and secondly how can time slots with penalty for untimely arrival be recognised in travel data. For the former an optimisation framework was given to derive the optimal time slots at the first and second stage with various options for penalty functions in case of a not timely arrival and various. For certain distribution functions of the handling and transportation times an explicit expression was derived. Also for the most complicated case, the second stage with propagation of the penalty of the first stage, an expression was derived. For the latter some insight was given to recognise these time slot constructions from correlation values of travel and handling times. Four cases were distinguished where each case showed specific characteristics in the correlation values. The characteristics could, in practice, be compensated by the interaction of humans.

\section{References}

1. Agra, A., Christiansen, M., Figueiredo, R., Hvattum, L.M., Poss, M., Requejo, C.: The robust vehicle routing problem with time windows. Computers \& operations research 40(3), 856-866 (2013)

2. de Armas, J., Melián-Batista, B., Moreno-Pérez, J.A., Brito, J.: Gvns for a realworld rich vehicle routing problem with time windows. Engineering Applications of Artificial Intelligence 42, 45-56 (2015)

3. Bushuev, M.A., Guiffrida, A.L.: Optimal position of supply chain delivery window: Concepts and general conditions. International Journal of Production Economics 137(2), 226-234 (2012)

4. Garg, D., Narahari, Y., Viswanadham, N.: Achieving sharp deliveries in supply chains through variance pool allocation. European Journal of Operational Research 171(1), 227-254 (2006)

5. Guiffrida, A.L., Nagi, R.: Cost characterizations of supply chain delivery performance. International journal of production economics 102(1), 22-36 (2006)

6. Safaei, M., Issa, S., Seifert, M., Thoben, K.D., Lang, W.: A method to estimate the accumulated delivery time uncertainty in supply networks. In: Dynamics in Logistics, pp. 337-347. Springer (2013)

7. Safaei, M., Mehrsai, A., Thoben, K.D.: A computational method in analyzing of delivery time uncertainty for highly complex supply networks. Measurement 55, 549-563 (2014)

8. Salani, M., Battarra, M., Gambardella, L.M.: Exact algorithms for the vehicle routing problem with soft time windows. In: Operations Research Proceedings 2014, pp. 481-486. Springer (2016)

9. Tanai, Y., Guiffrida, A.L.: Reducing the cost of untimely supply chain delivery performance for asymmetric laplace distributed delivery. Applied Mathematical Modelling 39(13), 3758-3770 (2015)

10. Vanany, I., Zailani, S., Pujawan, N.: Supply chain risk management: literature review and future research. IGI Global pp. 16-33 (2009) 